DIAS-STP-01-01

CERN-TH-2001-010

FAU-TP3-01-01

\title{
GAUGE INVARIANCE, BACKGROUND FIELDS AND MODIFIED WARD IDENTITIES
}

\author{
F. FREIRE \\ School of Theoretical Physics, Dublin Institute for Advanced Studies, \\ 10 Burlington Road, Dublin 4, Ireland \\ D. F. LITIM \\ Theory Division, CERN, \\ CH-1211 Geneva 23, Switzerland \\ J. M. PAWLOWSKI \\ Institut für Theoretische Physik III, Universität Erlangen-Nürnberg, \\ Staudtstraße 7, D-91058 Erlangen, Germany
}

\begin{abstract}
In this talk the gauge symmetry for Wilsonian flows in pure Yang-Mills theories is discussed. The background field formalism is used for the construction of a gauge invariant effective action. The symmetries of the effective action under gauge transformations for both the gauge field and the auxiliary background field are separately evaluated. Modified Ward-Takahashi and background field identities are used in my study. Finally it is shown how the symmetry properties of the full theory are restored in the limit where the cut-off is removed.
\end{abstract}

Introduction and aim: Wilsonian or Exact renormalisation group (ERG) equations have successfully been applied to non-perturbative phenomena in quantum field theories. Hence an ERG formulation of gauge theories is a promising tool for resolving open questions concerning the non-perturbative regime of these theories, e.g. confinement, chiral symmetry breaking. A key hurdle in such a task concerns the consistent and practicable introduction of an infra-red cut-off in a theory with a non-linear local symmetry.

In this talk $a^{a}$ will discuss this difficult matter in some detail. The quest can be presented as follow: How can it be ensured that a Wilsonian effective action shows the gauge symmetry of the underlying full theory? Let me first explain in more detail why this question requires a surgical look into. Implementations of the ERGl are most intricate when the symmetries of the theory are deformed by the intrinsic infra-red cut-off to this approach. As the integration of the flow equation is carried out it is necessary to guarantee that the information about the inherent symmetries of the theory is not washed out. In general, much work has been devoted to overcoming the involved problems mainly within different approaches to non-Abelian theories 目时团 Here, for the sake of clarity, I will discuss this problem for pure Yang-Mills theory. Moreover I use an approach to these theories within the background field formalism which permits the definition of a gauge invariant effective action.

${ }^{a}$ talk presented by FF at the Second Conference on the Exact Renormalization Group, Roma, Sep. 18-22, 2000. 
The aim is to clarify how it can be ensured that after the complete integration of the ERG equations, i.e. flow the cut-off scale $k$ to zero, a gauge invariant solution is obtained without the need of an extra fine-tuning. Furthermore, I will show that the resurgent symmetry does indeed correspond to the inherent gauge one. Most technical details will be bypassed in this presentation and may be found in a recent letter. 3

At the centre of my presentation is the quest of understanding how physical information is encrypted along the flow through an interplay between gauge invariance, Ward-Takahashi identities and background field identities. A similar programme has been pursued a few years ago in the context of Abelian theories. Background field identities were shown to contain the Ward identities under a requirement of gauge invariance.

The key ingredient of the present approach is the ERG equation for the effective action $\Gamma_{k}$. It describes the logarithmic rate of the change of $\Gamma_{k}$ with respect to the scale $k$. Following the standard implementation of the background field formalism I introduce a non-dynamical auxiliary field $\bar{A}$, the so-called background gauge field. Then formally the flow equation for pure Yang-Mills may be written as:

$$
\partial_{t} \Gamma_{k}\left[A, c, c^{*} ; \bar{A}\right]=\frac{1}{2} \operatorname{Tr}\left(\frac{\delta^{2} \Gamma_{k}}{\delta A \delta A}+R_{A}\right)^{-1} \partial_{t} R_{A}-\operatorname{Tr}\left(\frac{\delta^{2} \Gamma_{k}}{\delta c \delta c^{*}}+R_{C}\right)^{-1} \partial_{t} R_{C} .
$$

I use the common notation where $t=\ln k$ and the trace $\operatorname{Tr}$ denotes a sum over momenta, Lorentz and gauge group indices. The functions $R_{A}$ and $R_{C}$ implement the infra-red cut-off for the gauge field $A$ and ghost fields $c$ and $c^{*}$ respectively. They may also depend on the background field, to which I now turn your attention.

Background field formalism: I briefly summarise some important points about the background field formalism, in particular the rôle of different gauge transformations. The formalism is settled on the use of a background field dependent gauge-fixing condition that is invariant under a simultaneous gauge transformation of $\bar{A}$ and of the fields $A, c$ and $c^{*}$. This can be used for a definition of an effective action which is invariant under this combined gauge transformation. As $\bar{A}$ is involved in this transformation, the invariance of the effective action is, a priori, only an auxiliary symmetry. However, for the choice $\bar{A}=A$ it becomes the inherent gauge symmetry of the theory.

For a pure Yang-Mills theory including the ghost term,

$$
S=S_{\mathrm{cl}}+S_{\mathrm{gf}}+S_{\mathrm{gh}} .
$$

The classical action $S_{\mathrm{cl}}=\frac{1}{4} \int_{x} F_{\mu \nu}^{a} F_{\mu \nu}^{a}$ contains the field strength tensor $F_{\mu \nu}(A)=$ $\partial_{\mu} A_{\mu}-\partial_{\nu} A_{\mu}+g\left[A_{\mu}, A_{\nu}\right]$, where $F_{\mu \nu} \equiv F_{\mu \nu}^{a} t^{a}$ and $A_{\mu}=A_{\mu}^{a} t^{a}$ with the generators $t^{a}$ satisfying $\left[t^{a}, t^{b}\right]=f^{a b c} t^{c}$ and $\operatorname{tr} t^{a} t^{b}=-\frac{1}{2} \delta^{a b}$. I also use the shorthand notation $\int_{x} \equiv \int d^{d} x$. In the adjoint representation, the covariant derivative is

$$
D_{\mu}^{a b}(A)=\delta^{a b} \partial_{\mu}+g f^{a c b} A_{\mu}^{c} .
$$

The natural choice for the gauge fixing is the so-called background field gauge. The corresponding gauge-fixing and ghost actions are respectively,

$$
S_{\mathrm{gf}}=-\frac{1}{2 \xi} \int_{x}(A-\bar{A})_{\mu}^{a} \bar{D}_{\mu}^{a b} \bar{D}_{\nu}^{b c}(A-\bar{A})_{\nu}^{c}, \quad S_{\mathrm{gh}}=-\int_{x} c_{a}^{*} \bar{D}_{\mu}^{a c} D_{\mu}^{c d} c_{d},
$$


which involves the covariant derivative $\bar{D} \equiv D(\bar{A})$. The symmetries of the action in (2) can be inspected by two different gauge transformations. The first one gauge transforms the dynamic fields $A, c$, and $c^{*}$-it represents the underlying symmetry of the theory. Its infinitesimal generator $\mathcal{G}^{a}$ in a natural representation is defined as

$$
\mathcal{G}^{a}=D_{\mu}^{a b} \frac{\delta}{\delta A_{\mu}^{b}}-g f^{a b c}\left(c_{c} \frac{\delta}{\delta c_{b}}+c_{c}^{*} \frac{\delta}{\delta c_{b}^{*}}\right) .
$$

From the action of $\mathcal{G}^{a}$ on the fields it can be shown that $A$ transforms inhomogeneously, the ghosts transform as tensors and $\bar{A}$ is invariant. It follows that the covariant derivative transforms as a tensor.

The second transformation, denoted by the generated $\overline{\mathcal{G}}^{a}$, acts only on the background field,

$$
\overline{\mathcal{G}}^{a}=\bar{D}_{\mu}^{a b} \frac{\delta}{\delta \bar{A}_{\mu}^{b}}
$$

and under its action $\bar{A}$ transforms inhomogeneously like $A$ under $\mathcal{G}^{a}$, and therefore the covariant derivative $\bar{D}$ also transforms as a tensor. Note that the auxiliary transformation $\overline{\mathcal{G}}^{a}$ as it stands, does not carry any physical information.

I now turn your attention to the manner in which $\mathcal{G}^{a}$ and $\overline{\mathcal{G}}^{a}$ operate on the action $S$. The classical action is invariant under both transformations since it does not depend on the background field, while neither $S_{\mathrm{gf}}$ nor $S_{\mathrm{gh}}$ are invariant under (5) or (6). For (5) it follows,

$$
\mathcal{G}^{a}(x) S_{\mathrm{gf}}=\frac{1}{\xi} D_{\mu}^{a b} \bar{D}_{\mu}^{b c} \bar{D}_{\nu}^{c d}(A-\bar{A})_{\nu}^{d}(x), \quad \mathcal{G}^{a}(x) S_{\mathrm{gh}}=f^{b d c} \bar{D}_{\mu}^{a d}\left(c_{b}^{*} D_{\mu}^{c e} c_{e}\right) .
$$

Now from the explicit expression for $S_{\mathrm{gf}}$ and $S_{\mathrm{gh}}$, as given in (4), it follows that (7) just displays $-\overline{\mathcal{G}}^{a} S_{\text {gf }}$ and $-\overline{\mathcal{G}}^{a} S_{\mathrm{gh}}$ respectively. Thus, in the background field gauge, each term in the Yang-Mills action (2) is separately invariant under the combined transformation $\mathcal{G}+\overline{\mathcal{G}}$. A key point of the background field formalism has been reached: the action resulting from setting the background field equal to the gauge field, i.e. $\hat{S}\left[A, c, c^{*}\right] \equiv S\left[A, c, c^{*} ; \bar{A}=A\right]$ is invariant under the physical symmetry generated by (5), $\mathcal{G}^{a} \hat{S}\left[A, c, c^{*}\right]=0$, with $S\left[A, c, c^{*} ; \bar{A}\right]$ satisfying the classical 'Ward Takahashi identity', $\mathcal{G}^{a} S=\mathcal{G}^{a}\left(S_{\mathrm{gf}}+S_{\mathrm{gh}}\right)$.

At quantum level this symmetry turns into the gauge invariance of the effective action $\Gamma\left[A, c, c^{*} ; \bar{A}=A\right]$, which in turn satisfies the Ward-Takahashi identity for a non-Abelian gauge theory. However I remind you that it is only the combination of both statements that gives a physical meaning to this gauge invariance. Note that heuristically this result stems from the observation that in the quantised theory the source only couple to the fluctuation field

$$
a_{\mu}^{a}=A_{\mu}^{a}-\bar{A}_{\mu}^{a},
$$

and the gauge fixing condition (4) only constrains $a_{\mu}^{a}$. 
Background field dependent Wilsonian flows: In order to effectively implement the background field formalism for the coarse-grained effective Yang-Mills action I choose the regulator terms

$$
\Delta S_{k}=\Delta S_{k, A}+\Delta S_{k, C},
$$

for the gauge and the ghost fields, respectively, to be 3

$$
\begin{aligned}
\Delta S_{k, A} & =\frac{1}{2} \int_{x}(A-\bar{A})_{\mu}^{a} R_{A}^{a b}\left(P_{A}^{2}\right)(A-\bar{A})_{\nu}^{b} \\
\Delta S_{k, C} & =\int_{x} c_{a}^{*} R_{C}^{a b}\left(P_{C}^{2}\right) c_{b} .
\end{aligned}
$$

The arguments $P_{A}^{2}$ and $P_{C}^{2}$ of the regulator functions are appropriately defined background field dependent Laplaceans, and their choice might determine the symmetries of the resulting theory. For instance, in order to have an action $S_{k}$ which is gauge invariant under the combined transformation it is only necessary to required that both $P_{A}^{2}$ and $P_{C}^{2}$ transform as tensors under $\mathcal{G}+\overline{\mathcal{G}}$. Thus, in the following, I shall assume that such a choice has been made.

Up to this point, I have restricted the presentation to the classical action with the regulator terms (9) added. The computation of the coarse-grained effective action $\Gamma_{k}$ follows the usual procedure. Consider the Schwinger functional $W_{k} \equiv$ $W_{k}\left[J_{\mu}, \eta, \eta^{*} ; \bar{A}_{\mu}\right]$,

$$
\exp W_{k}=\int \prod_{a}\left\{\mathcal{D} A_{\mu}^{a} \mathcal{D} c_{a} \mathcal{D} c_{a}^{*}\right\} \exp \left[-S_{k}+\int\left(J_{\mu}^{a}(A-\bar{A})_{\mu}^{a}+\eta_{a}^{*} c_{a}-c_{a}^{*} \eta_{a}\right)\right] .
$$

where $\left(J, \eta, \eta^{*}\right)$ are the respective sources. Then the effective action $\Gamma_{k}$ is given by

$$
\begin{aligned}
\Gamma_{k}\left[A, c, c^{*} ; \bar{A}\right]=-W_{k}\left[J, \eta, \eta^{*} ; \bar{A}\right] & -\Delta S_{k}\left[A, c, c^{*} ; \bar{A}\right] \\
& +\int_{x}\left(J_{\mu}^{a}(A-\bar{A})_{\mu}^{a}+\eta_{a}^{*} \bar{c}_{a}-c_{a}^{*} \eta_{a}\right) .
\end{aligned}
$$

The flow equation for $\Gamma_{k}$ has already been given at the beginning, (11). Now I introduce the effective action $\hat{\Gamma}_{k}$,

$$
\hat{\Gamma}_{k}\left[A, c, c^{*}\right] \equiv \Gamma_{k}\left[A, c, c^{*} ; \bar{A}=A\right] .
$$

As I shall argue later, this new action is gauge-invariant. Its flow equation, of course, is given by the flow of $\Gamma_{k}$ in (11), but evaluated at $\bar{A}=A$. It is important to stress that $\partial_{t} \hat{\Gamma}_{k}$, since it depends on the second functional derivatives of $\Gamma_{k}$ with respect to the dynamical fields (at $\bar{A}=A$ ), is a functional of $\Gamma_{k}$ and not a functional of $\hat{\Gamma}_{k}$. This means that is not sufficient to study the symmetries of $\hat{\Gamma}_{k}$ but also necessary to study those of $\Gamma_{k}$.

Modified and background field Ward-Takahashi identities: I will now discuss the Ward-Takahashi identities that are related to the gauge transformations (5) and (6). In the Wilsonian formalism, due to the presence of a coarse-graining, these identities receive a contribution from the regulator term. The identity that follows 
from considering $\mathcal{G}^{a} \Gamma_{k}$ is called the modified Ward-Takahashi identities (mWI). A second identity follows from $\overline{\mathcal{G}}^{a} \Gamma_{k}$ and I shall denote it as the background field Ward-Takahashi identities (bWI).

As $S_{k}$ is invariant under the action of $\mathcal{G}^{a}+\overline{\mathcal{G}}^{a}$ it can be read off from the Schwinger functional (12) and the effective action (13), that $\mathcal{G}^{a}+\overline{\mathcal{G}}^{a}$ leaves the functional $\Gamma_{k}$ invariant for generic $A$ and $\bar{A}$ configurations,

$$
\left(\mathcal{G}^{a}+\overline{\mathcal{G}}^{a}\right) \Gamma_{k}=0 .
$$

Therefore by requiring $A=\bar{A}, \hat{\Gamma}_{k}$ is also invariant, $\mathcal{G}^{a} \hat{\Gamma}_{k}=0$, which for $k=0$ expresses the desired physical gauge invariance. Consequently, for $k \neq 0$, physical gauge invariance is encoded in the behaviour of $\Gamma_{k}$ under the transformation $\mathcal{G}^{a}$. I emphasise again that, in order to attain this crucial result, I had to keep track of the effects from the transformations $\mathcal{G}^{a}$ and $\overline{\mathcal{G}}^{a}$ on $\Gamma_{k}$ separately.

For pure Yang-Mills theories the mWI is given by

$$
\mathcal{G}^{a}(x) \Gamma_{k}=\mathcal{G}^{a}(x)\left(S_{\mathrm{gf}}+S_{\mathrm{gh}}\right)+L_{k}^{a}(x)+L_{R, k}^{a}(x) .
$$

Both $L_{k}$ and $L_{R, k}$ display loop terms. The first term $L_{k}$ stands for the well-known loop contributions to Ward-Takahashi identities in non-Abelian gauge theories originating from $\left\langle\mathcal{G}^{a}\left(S_{\mathrm{gf}}+S_{\mathrm{gh}}\right)\right\rangle_{J}$, whilst the second term is due to the regulator terms and clearly vanishes when $k \rightarrow 0$. It follows that the mWI (16) turns into the standard WI for $k=0$,

$$
\mathcal{G}^{a} \Gamma=L_{0}^{a}
$$

As for the bWI, by applying $\overline{\mathcal{G}}^{a}$ to $W_{k}[J, \eta, \bar{\eta} ; \bar{A}]$ it follows from (12) and (13) after some manipulations that the effective action $\Gamma_{k}$ obeys the equation

$$
\overline{\mathcal{G}}^{a} \Gamma_{k}=\overline{\mathcal{G}}^{a}\left(S_{\mathrm{gf}}+S_{\mathrm{gh}}\right)-\left(L_{k}^{a}+L_{R, k}^{a}\right) .
$$

The combined gauge invariance of $\Gamma_{k}$, Eq. (15), follows immediately from this identity and the mWI given by (16).

Symmetries of the flow and physical gauge invariance: The implementation of a coarsegraining modifies the gauge symmetry of the theory as I mentioned before. At the formal level it is clear that the original symmetry is restored when the coarsegraining scale is removed. A more delicate problem is to guarantee that this also happens at the level of the solution to the flow equation.

To understand how gauge invariance is encoded throughout the flow, it is pivotal to also study the action of the symmetry transformations on $\partial_{t} \Gamma_{k}$, Eq, (1D). Under the combined gauge transformation the flow of $\Gamma_{k}$ transforms as

$$
\left(\mathcal{G}^{a}+\overline{\mathcal{G}}^{a}\right) \partial_{t} \Gamma_{k}=0 .
$$

An immediate consequence is that $\mathcal{G}^{a} \partial_{t} \hat{\Gamma}_{k}=0$. Note that the only input for (19) was the invariance of $\Gamma_{k}$. Thus, when the initial effective action $\Gamma_{\Lambda}$ is invariant under $\mathcal{G}^{a}+\overline{\mathcal{G}}^{a}$, it follows that the full effective action $\Gamma_{0}$ is also invariant, $\left(\mathcal{G}^{a}+\overline{\mathcal{G}}^{a}\right) \Gamma_{0}=$ 0 . In other words, (15) and (19) are the proof that the flow and the combined 
transformation commute. Moreover, $\Gamma_{0}$ satisfies the usual WI (17). This means that the line of arguments for the background field formalism can be followed here as well. Hence the equation $\mathcal{G}^{a} \hat{\Gamma}_{0}=0$ displays physical gauge invariance.

Now I wish to make a final remark on the consistency of the mWI 16 with the flow. Here, as in other formulations of Wilsonian flows in gauge theories, flow of the mWI is proportional to itself. Such common property states that if the effective action $\Gamma_{k}$ satisfies the mWI at some scale $k, e . g$ the initial one $k=\Lambda$, then $\Gamma_{k}$ automatically satisfies the mWI at any scale $k$, provided it is obtained from integrating the flow equation, and in particular, $\Gamma_{0}$ satisfies the usual Ward-Takahashi identity.

Summary of the Talk: I have established a complete set of equations relevant for the control of gauge invariance in the ERG approach to pure Yang-Mills theories. In particular, it can be inferred that there is no requirement for additional fine-tuning conditions, despite the presence of a background field. Moreover, I have shown that invariance of the effective action under a combined gauge transformation of all fields follows from mWI and bWI. More generally, two of these three properties of the effective action (invariance, $\mathrm{mWI}$ and $\mathrm{bWI}$ ) lead to the third one.

Consequently, a key property of the usual background field formalism is maintained in the ERG approach: by virtue of the auxiliary background identity it follows that physical gauge invariance is reflected in the invariance of the effective action under the combined gauge transformation of all fields.

The formalism is not only suitable for formal or analytic analysis, but also, it elucidates the problem of how to filter the contribution of spurious unphysical modes in a numerical computation of approximate solutions to flow equations in gauge theories.

\section{Acknowledgements}

FF thanks the organisers for their financial support.

\section{References}

1. J. Polchinski, Nucl. Phys. B231 (1984) 269.

2. F. Freire, D. F. Litim and J. M. Pawlowski, Phys. Lett. B495 (2000) 256 hep-th/0009110].

3. M. Reuter and C. Wetterich, Nucl. Phys. B417 (1994) 181.

4. U. Ellwanger, Phys. Lett. B335 (1994) 364 hep-th/9402077]. U. Ellwanger, M. Hirsch and A. Weber, Z. Phys. C69 (1996) 687 [hep-th/9506019]; Eur. Phys. J. C1 (1998) 563 hep-ph/9606468.

5. F. Freire and C. Wetterich, Phys. Lett. B380 (1996) 337 [hep-th/9601081];

6. T. R. Morris, Nucl. Phys. B573 (2000) 97 hep-th/9910058; hep-th/0006064.

7. D.F. Litim and J.M. Pawlowski, Proceedings of the Workshop on the ERG in Faro, Portugal, Sep98, published in World Scientific hep-th/9901063.

8. L.F. Abbott, Nucl. Phys. B185 (1981) 189. 